\title{
Vitamin E Level Changes in Serum and Red Blood Cells Due to Acute Exhaustive Exercise in Collegiate Women
}

\author{
Yukari KawaI, ${ }^{1, *}$ Teruichi Shimomitsu, ${ }^{1}$ Yoshikazu Takanami, ${ }^{1}$ Norio Murase, ${ }^{1}$ \\ Toshihito KATSUMURA ${ }^{1}$ and Chizuko MARUYAma ${ }^{2}$ \\ ${ }^{1}$ Department of Preventive Medicine and Public Health, Tokyo Medical University, Tokyo 160-8402, Japan \\ ${ }^{2}$ Department of Food and Nutrition, Japan Women's University, Tokyo 112-0015, Japan
}

(Received November 4, 1999)

\begin{abstract}
Summary The purpose of this study was to investigate the change in vitamin E level in both serum and red blood cells (RBC) during exercise and to clarify the effect of vitamin E supplementation. Ten young sedentary female subjects received $200 \mathrm{mg}$ D- $\alpha$-tocopherol acetate daily for $1 \mathrm{wk}$ after the initial exercise bout. After $1 \mathrm{wk}$ of vitamin E supplementation, the same subjects repeated the same exercise. Before vitamin E supplementation, the $\alpha$-tocopherol level in the serum (serum- $\alpha$-tocopherol) did not change after exercise, but a significant decrease in the $\alpha$-tocopherol level in RBC (RBC- $\alpha$-tocopherol) was observed after exercise $(p<0.05)$. On the other hand, after vitamin E supplementation, the serum- $\alpha$-tocopherol level decreased significantly after exercise $(p<0.05)$, while the RBC- $\alpha$-tocopherol level was maintained after exercise. Furthermore, a negative correlation between the changes in serum- and RBC- $\alpha$-tocopherol levels was observed only after vitamin E supplementation $(r=0.667, p<0.05)$. The present results suggest that as RBC suffers oxidative stress, vitamin $\mathrm{E}$ in $\mathrm{RBC}$ is consumed to protect $\mathrm{RBC}$ from oxidative damage during exercise. These results also suggest that when there is a sufficient amount of vitamin $\mathrm{E}$ in the serum, vitamin $\mathrm{E}$ is shifted from the serum to RBC, resulting in a steady RBC- $\alpha$-tocopherol level and a decrease in the serum- $\alpha$-tocopherol level under oxidative stress such as exercise.

Key Words $\alpha$-tocopherol, exercise, vitamin E supplementation, oxidative stress, red blood cells
\end{abstract}

Exercise enhances oxidative metabolism, and it is thereby believed that exercise increases the generation of reactive oxidative species (ROS) and lipid peroxidation (1). In a previous study, Szczesniak et al. (2) reported that RBC-glutathione content was reduced and serum thiobarbituric acid reactive substances (TBARS) were increased during maximal treadmill exercise. Oostenbrug et al. (3) reported that decreased RBC deformability, which causes the consumption of plasma antioxidants, appeared during endurance exercise. The protective effects of dietary antioxidants against the production of lipid peroxide during exercise have received much attention recently. Vitamin $\mathrm{E}$ is well accepted as the most effective lipid-soluble, chain-breaking antioxidant present in nature that protects lipids from peroxidation in cell membranes and serum (4). Our previous report indicated that vitamin E pools can be redistributed among tissues; therefore, exercise may enhance such a transfer in the prevention of oxidative damage (5). However, there are few reports regarding the physiological movement of vitamin E among tissues under exercise-induced oxidative stress (6).

The changes of vitamin E level in serum and tissues suffering from oxidative stress must be examined in order to clarify the role of vitamin $\mathrm{E}$ in the antioxidant system enhanced by exercise. However, a human study

*E-mail: kawai-y@tokyo-med.ac.jp based on such an idea has not yet been performed until now. Therefore, the purpose of this study was to investigate the change in vitamin $\mathrm{E}$ in blood following a single bout of short, intensive exercise. It is known that there is an increase in the production of ROS through the increase in the rate of auto-oxidation of hemoglobin (7), which occurs when RBC carry oxygen. Therefore, the vitamin $\mathrm{E}$ level in RBC was determined in this study, because RBC are thought to suffer from oxidative stress during exercise. In addition, we also examined the effect of vitamin $\mathrm{E}$ supplementation on these changes.

\section{MATERIALS AND METHODS}

Subjects. Ten sedentary collegiate females were used as the subjects in this study. The characteristics of the subjects are summarized in Table 1 . They were all healthy nonsmokers, and were able to maintain a regular diet and a constant level of physical activity throughout the study. Subjects gave informed written consent to participate in this study. This experiment was performed in accordance with the principles of the Declaration of Helsinki of the World Medical Association.

Study design. The study design is shown in Fig. 1. The subjects were instructed to refrain from strenuous physical exercise on the day before performing maximal exercise. On the day of the exercise, subjects performed an incremental cycle ergometry test to exhaustion 
(maximal exercise). Blood samples were collected before and immediately after the maximal exercise. The subjects were further given a $200 \mathrm{mg}$ supplement of vitamin E; they received two bottles ( $100 \mathrm{mg}$ of D- $\alpha$-tocopherol acetate in $20 \mathrm{~mL}$ soybean lecithin emulsion (Eisai Co., Ltd., Tokyo, Japan) twice daily for $1 \mathrm{wk}$. The supplement regimen was determined by the measurement of each subject's serum- $\alpha$-tocopherol before and after the supplementation period. After $1 \mathrm{wk}$ of vitamin E supplementation, subjects repeated the same maximal exercise as described above. All tests were conducted during the same time of the day to minimize the effect of the circadian rhythm and hormonal cycle. To minimize the influence of the menstrual cycle, we adjusted the testing date for each subject to match and synchronize the menstrual phase.

Maximal exercise. The maximal exercise protocol is shown in Fig. 2. Exercise tests were performed on a Siemens cycle ergometer (Germany). Following a warm-up stage at 0 watt for $2 \mathrm{~min}$, exercise power output was increased at a rate of 20 watts/min until the

Table 1. Characteristics of subjects.

\begin{tabular}{lr}
\hline Number & 10 \\
Sex & Female \\
Age $(\mathrm{y})$ & $21.2 \pm 0.5$ \\
Body height $(\mathrm{cm})$ & $158.7 \pm 1.4$ \\
Body weight $(\mathrm{kg})$ & $49.8 \pm 1.4$ \\
Body mass index $\left(\mathrm{kg} / \mathrm{m}^{2}\right)$ & $19.9 \pm 0.4$ \\
$\dot{\mathrm{V}} \mathrm{O}_{2} \max (\mathrm{mL} / \mathrm{kg} / \mathrm{min})$ & $28.3 \pm 1.2$ \\
\hline
\end{tabular}

Values are means \pm SE. subjects were unable to maintain the pedaling frequency. During continuous progressive cycling exercise, subjects maintained a constant pedaling rate of $60 \mathrm{rpm}$, which was set using a metronome. Heart rate and electrocardiogram were recorded once every minute by a Model CASE 12, Marquette (USA), and blood pressure was recorded once every minute using a Model STBP680, Nippon Colin (Japan). Respiratory gas exchange parameters were measured on-line with standard open-circuit techniques using System 2001, Medical Graphics Co. (USA). The maximal oxygen uptake $\left(\dot{\mathrm{V}} \mathrm{O}_{2} \mathrm{max}\right)$ of each subject was determined.

Sample analysis. Blood samples were collected by venipuncture and were centrifuged at $2,000 \times g$ for 10 min to separate the serum. The obtained serum was used to measure $\alpha$-tocopherol and total cholesterol. The samples were frozen at $-110^{\circ} \mathrm{C}$ under $\mathrm{N}_{2}$ gas until the analysis was completed.

Serum total cholesterol was measured using the method of Richmond (8). Serum- $\alpha$-tocopherol was measured by a modified method of Abe and Katsui (9) using high-performance liquid chromatography (HPLC). $\alpha$-Tocopherol was extracted from $0.5 \mathrm{~mL}$ of serum with $5.0 \mathrm{~mL}$ of $n$-hexane after the precipitation of proteins with ethanol. Four milliliters of the $n$-hexane extract was evaporated under $\mathrm{N}_{2}$ gas, and the residue was dissolved in $1.0 \mathrm{~mL}$ of 2,2,5,7,8-pentamethyl-6-hydroxychroman (PMC) $(1.5 \mu \mathrm{g} / \mathrm{mL})$ in ethanol. The chromatographic conditions used with a fluorometric detector, Shimazu RF-535 (Shimazu Co., Japan), were as follows: column, Shim-pack CLC-NH $\mathrm{NH}_{2}(6.0$ i.d $\times$ $150 \mathrm{~mm}$ ) (Shimazu Co., Japan); mobile phase, $n$-hexane:

Before vitamin E supplementation

After vitamin E supplementation

\begin{tabular}{c|c|c|c|}
\cline { 2 - 3 } & Max. Ex. & Max. Ex. \\
\hline & Blood draw & $200 \mathrm{mg} / \mathrm{d}$ of $\mathrm{D}$ - $\alpha$-tocopherol acetate $\rightarrow$ & onk
\end{tabular}

Fig. 1. Study design. Subjects consisted of ten collegiate females. Subjects performed the first graded exercise to exhausion by cycle ergometer (maximal exercise). The same subjects took vitamin E supplement for $1 \mathrm{wk}$ and then performed the second maximal exercise. Blood samples were taken before and immediately after exercise. Max. Ex., maximal exercise.

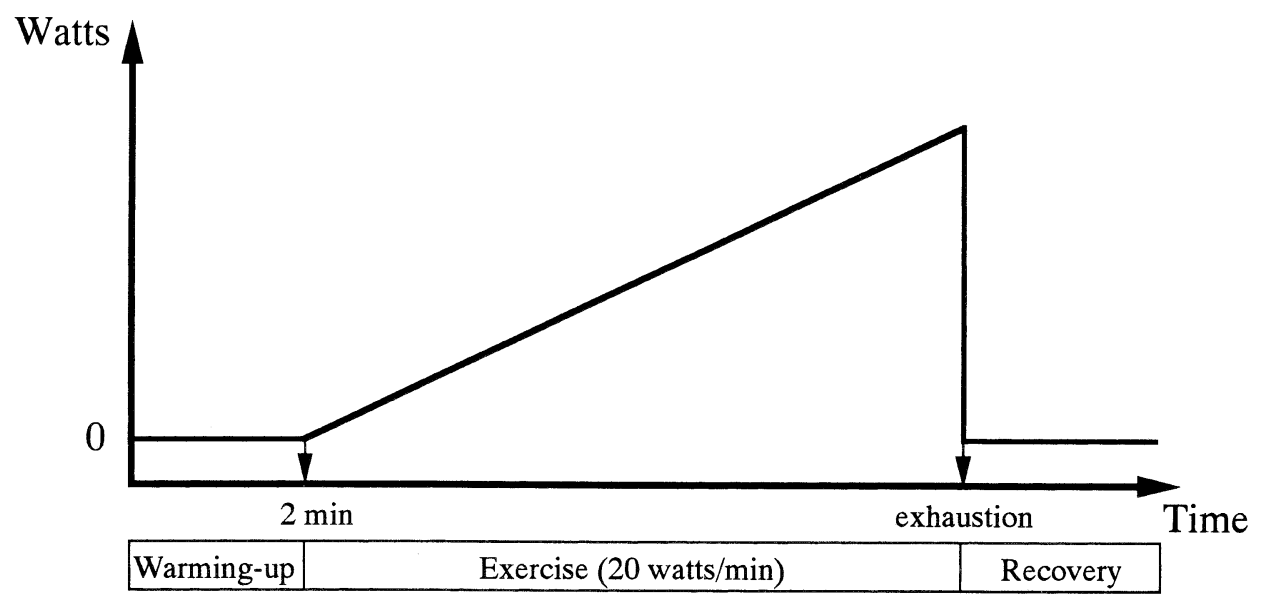

Fig. 2. Maximal exercise protocol. All subjects performed incremental cycle ergometry to exhaustion. After warmimg-up ( 0 watt, $2 \mathrm{~min}$ ), the work rate was increased to a rate of 20 watts $/ \mathrm{min}$, while the pedaling rate remained constant at $60 \mathrm{rpm}$. 
2-propanol (100:2.8, v/v); flow rate, $1.5 \mathrm{~mL} / \mathrm{min}$; and injection volume, $10 \mu \mathrm{L}$.

RBC- $\alpha$-tocopherol was measured in blood samples collected into tubes containing heparin on the same day or the next day. The collected fresh venous blood was transferred into centrifuge tubes containing 1\% EDTA$2 \mathrm{Na} / 0.9 \% \mathrm{NaCl}$ to obtain $2 \%$ whole blood. The blood was then centrifuged at $850 \times g$ for $5 \mathrm{~min}$, and the plasma and buffy coat were removed. The RBC were washed three times with 5-fold volumes of an isotonic phosphate-buffered $\mathrm{NaCl}$ solution containing EDTA. After the third washing, the hematocrit of the RBC was made up to about $50 \%$, and then the correct hematocrit was measured. RBC- $\alpha$-tocopherol was extracted using a modified method of Hatam and Kaydan. (10). Washed RBC $(0.4 \mathrm{~mL})$ with a known hematocrit were mixed with $1.0 \mathrm{~mL}$ of $6 \%$ pyrogallol in ethanol and $1.0 \mathrm{~mL}$ of PMC $(0.3 \mu \mathrm{g} / \mathrm{mL})$ in ethanol (an internal standard). After thorough mixing, the samples were heated for $2 \mathrm{~min}$ in a $70^{\circ} \mathrm{C}$ water bath. Following that, $0.2 \mathrm{~mL}$ of $60 \% \mathrm{KOH}$ was added, and the samples were heated at $70^{\circ} \mathrm{C}$ for $30 \mathrm{~min}$. After saponification, the samples were cooled in an ice bath. The saponified material was extracted at room temperature after the addition of $4.5 \mathrm{~mL}$ of $1 \% \mathrm{NaCl}$ and then $3 \mathrm{~mL}$ of a mixture of ethyl acetate and $n$-hexane $(1: 9, \mathrm{v} / \mathrm{v})$. The samples were shaken vigorously for $5 \mathrm{~min}$ by a shaker. The samples were centrifuged at $2,000 \times g$ for $5 \mathrm{~min}$ to ensure complete phase separation. Two milliliters of the $n$-hexane phase (upper layer) was mixed with $1.0 \mathrm{~mL}$ of decane $(50 \mathrm{mg} / \mathrm{mL})$ in $n$-hexane. These samples were evaporated under $\mathrm{N}_{2}$ gas. After evaporation, $0.1 \mathrm{~mL}$ of $n$ hexane was added to the samples and then $\alpha$-tocopherol in the prepared samples was measured by HPLC. RBC- $\alpha$-tocopherol was also measured using HPLC under the same conditions as used for the measurement of serum- $\alpha$-tocopherol.

Plasma lactate was measured using a Model 2300 YSI Glucose/Lactate Analyzer (Yellow Springs Instrument Co., Inc., USA). Blood samples containing EDTA-2K were used to measure hemoglobin concentration and hematocrit for estimation of the change in plasma volume through exercise (11). Data taken immediately after exercise were normalized according to the change in plasma volume through exercise.

Statistical analysis. All results are expressed as mean \pm SE. Statistical analysis was undertaken using repeated measures ANOVA. The difference with a $p$ value less than 0.05 was considered significant.

\section{RESULTS}

Effects of vitamin E supplementation on exercise parameter

As shown in Table 2, exercise duration, $\dot{\mathrm{V}} \mathrm{O}_{2} \max$ and maximal heart rate did not change before and after vitamin E supplementation. There was no difference in the post-exercise plasma concentration before and after vitamin E supplementation. Unless otherwise noted, the following results were obtained immediately after exercise. A markedly large increase in the level of plasma lactate was observed after maximal exercise before and after vitamin E supplementation (Table 3). Furthermore, the percent change in the plasma lactate level did not differ before and after vitamin E supplementation (change in plasma lactate level: before vitamin E supplementation, $739.6 \pm 269.0 \%$; after vitamin $\mathrm{E}$ supplementation, $717.8 \pm 443.2 \%$ ).

Effects of vitamin $E$ supplementation on $\alpha$-tocopherol levels

The results in Table 3 show the serum- and RBC- $\alpha$ tocopherol levels obtained before and after maximal exercise in subjects before and after vitamin E supplementation. Serum- and RBC- $\alpha$-tocopherol concentrations were $9.54 \pm 0.87$ and $2.00 \pm 0.16 \mu \mathrm{g} / \mathrm{mL}$ packed cells, respectively, before exercise and vitamin E supplementation. After 1 wk of vitamin E supplementation, serum-

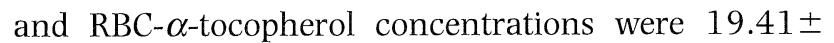
1.56 and $3.75 \pm 0.38 \mu \mathrm{g} / \mathrm{mL}$ packed cells , respectively, before exercise. Both serum- and RBC- $\alpha$-tocopherol levels were increased 2 -fold by vitamin $\mathrm{E}$ supplementation $(p<0.01)$.

Before vitamin E supplementation, the serum- $\alpha$-tocopherol level did not changed significantly by exercise $(9.54 \pm 0.87 \mu \mathrm{g} / \mathrm{mL}$ before exercise vs. $8.72 \pm 0.74 \mu \mathrm{g} /$ $\mathrm{mL}$ after exercise). However, the RBC- $\alpha$-tocopherol level was significantly decreased by exercise $(2.00 \pm 0.16 \mu \mathrm{g} /$ $\mathrm{mL}$ packed cells before exercise vs. $1.80 \pm 0.12 \mu \mathrm{g} / \mathrm{mL}$ packed cells after exercise $)(p<0.05)$.

Ater vitamin E supplementation, a significant decrease in the serum- $\alpha$-tocopherol level by exercise was

Table 2. Changes in indices of maximal exercise in female subjects before and after vitamin E supplementation.

Vitamin E supplementation

Indices

\begin{tabular}{cc}
\multicolumn{1}{c}{ Before } & \multicolumn{1}{c}{ After } \\
$9 \min 04 \pm 23 \mathrm{~s}$ & $9 \min 13 \pm 24 \mathrm{~s}$ \\
$28.3 \pm 1.2$ & $29.1 \pm 1.4$ \\
$176.0 \pm 2.6$ & $178.2 \pm 2.9$ \\
$233.3 \pm 7.7$ & $234.0 \pm 7.0$ \\
$83.9 \pm 1.5$ & $83.8 \pm 2.5$
\end{tabular}

Values are means \pm SE.

Max. Ex., maximal exercise; $\dot{\mathrm{V}} \mathrm{O}_{2}$ max, maximal oxygen uptake; HRmax, maximal heart rate.

${ }^{1}$ Values are rate of changes in heart rate from before exercise to immediately after exercise.

No statistically significant differences were found between the 1 st and 2 nd exercises. 
Table 3. Serum- and RBC- $\alpha$-tocopherol levels and serum lactate level before and after exercise in female subjects before and after vitamin E supplementation.

\begin{tabular}{lcc}
\hline & Before exercise & After exercise \\
\hline Serum-lactate $(\mathrm{mg} / \mathrm{dL})$ & & $63.3 \pm 6.2^{\mathrm{a}}$ \\
$\quad$ before vitamin E supplementation & $9.1 \pm 0.8$ & $66.8 \pm 7.2^{\mathrm{a}}$ \\
$\quad$ after vitamin E supplementation & $14.6 \pm 4.3$ & $8.72 \pm 0.74$ \\
Serum- $\alpha$-tocopherol $(\mu \mathrm{g} / \mathrm{dL})$ & & $17.04 \pm 1.47^{\mathrm{b}}$ \\
$\quad$ before vitamin E supplementation & $9.54 \pm 0.87$ & $4.35 \pm 0.34$ \\
$\quad$ after vitamin E supplementation & $19.41 \pm 1.56^{\mathrm{c}}$ & $8.67 \pm 0.75^{\mathrm{b}}$ \\
$\quad$ Serum- $\alpha$-tocopherol/total-cholestrol & & \\
$\quad$ before vitamin E supplementation & $5.32 \pm 0.47$ & $1.80 \pm 0.12^{\mathrm{b}}$ \\
$\quad$ after vitamin E supplementation & $11.33 \pm 0.93^{\mathrm{c}}$ & $3.60 \pm 0.41$
\end{tabular}

Values are means \pm SE.

${ }^{\mathrm{a}} p<0.01$ compared to values obtained before exercise; ${ }^{\mathrm{b}} p<0.05$ compared to values obtained before exercise; ${ }^{c} p<0.01$ compared to values obtained before vitamin E supplementation.
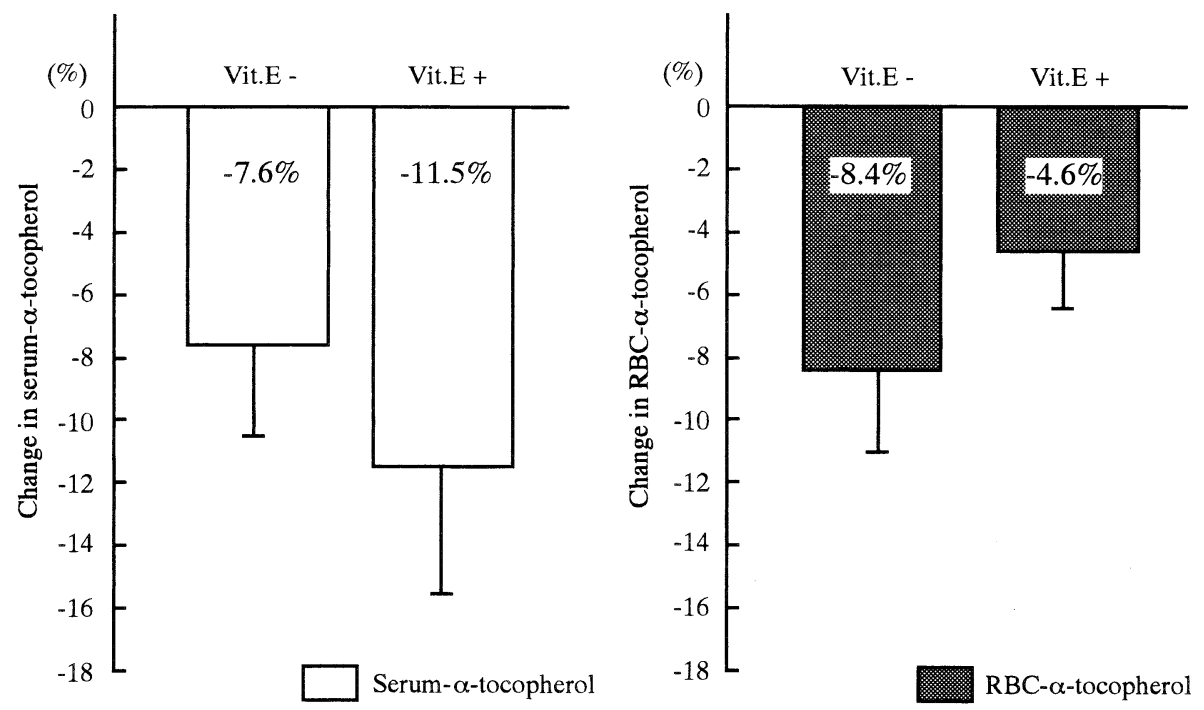

Fig. 3. Changes in serum- and RBC- $\alpha$-tocopherol levels before and after exercise in female subjects before and after vitamin E supplementation. The decrease in serum- $\alpha$-tocopherol level by exercise after vitamin E supplementation was larger than that before vitamin E supplementation. However, the decrease in RBC- $\alpha$-tocopherol level by exercise after vitamin E supplementation was less than that before vitamin E supplementation.

Mean \pm SE, Vit.E- , before vitamin E supplementation; Vit.E+, after vitamin E supplementation.

observed $(19.41 \pm 1.56 \mu \mathrm{g} / \mathrm{mL}$ before exercise vs. $17.04 \pm 1.47 \mu \mathrm{g} / \mathrm{mL}$ after exercise $)(p<0.05)$. However, the RBC- $\alpha$-tocopherol level was not changed significantly by exercise $(3.75 \pm 0.38 \mu \mathrm{g} / \mathrm{mL}$ packed cells before exercise vs. $3.60 \pm 0.41 \mu \mathrm{g} / \mathrm{mL}$ packed cells after exercise). The average decrease of serum- $\alpha$-tocopherol level by exercise after vitamin E supplementation was larger than that before vitamin E supplementation. The average decline of RBC- $\alpha$-tocopherol level was significantly decreased by exercise before vitamin E supplementation, although there was no significant change in the RBC- $\alpha$-tocopherol level after vitamin E supplementation (Fig. 3). There was a significant negative correlation between the changes in serum- and RBC- $\alpha$-tocopherol levels by exercise only after vitamin E supplementation ( $r=0.667, p<0.05)$ (Fig. 4).

\section{DISCUSSION}

It is believed that exercise causes an increase in the generation of ROS through the production of energy from the mitochondria (12) and transient ischemiareperfusion that may occur in the muscle and joints (13). In several reports, vitamin E supplementation reduced the increased level of serum TBARS, an index of lipid peroxidation, which was caused by exercise-induced oxidative damage $(14,15)$. Furthermore, an increase in the rate of auto-oxidation of hemoglobin is known to occur when RBC carry oxygen due to an increase in the oxygen uptake during exercise (7). Such an increase in the rate of auto-oxidation of hemoglobin during exercise may enhance oxidative stress. Therefore, RBC may suffer greatly from oxidative stress dur- 
Before vitamin E supplementation

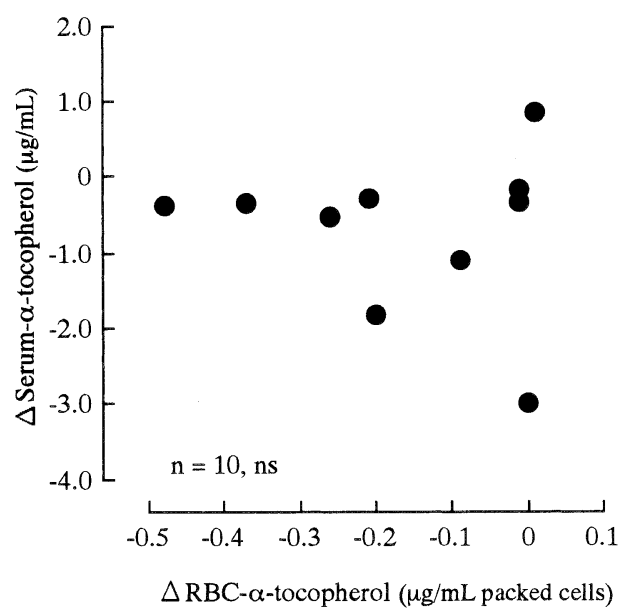

After vitamin E supplementation

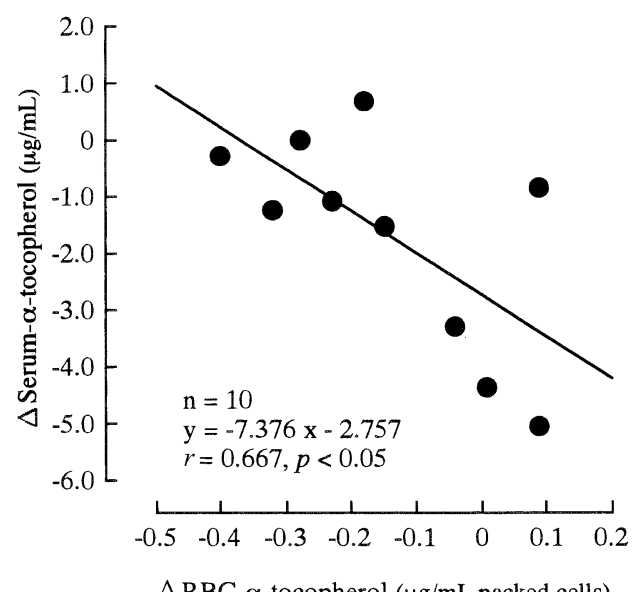

Fig. 4. Correlation between the changes in serum- and RBC- $\alpha$-tocopherol levels before and after exercise in female subjects before and after vitamin E supplementation. Before vitamin E supplementation, the change in serum- $\alpha$-tocopherol level was not correlated with that of RBC- $\alpha$-tocopherol level (left). But changes in serum- and RBC- $\alpha$-tocopherol levels were significantly correlated after vitamin E supplementation $(r=0.667, p<0.05)$ (right).

ing exercise. Since vitamin $\mathrm{E}$ is the most potent lipid-soluble antioxidant in the RBC membrane, it is important to examine the change in the vitamin E level of RBC following exercise in order to clarify the role of vitamin $\mathrm{E}$ as an antioxidant in the cell membrane during exercise. However, there are only a few reports concerning the change of vitamin $\mathrm{E}$ level in $\mathrm{RBC}$ following exercise. Duthie et al. (16) demonstrated that the RBC- $\alpha$-tocopherol level showed a significant increase after completion of a half-marathon. They speculated that it might be caused by the mobilization of vitamin E from yet unidentified stores. We previously reported that serumand RBC- $\alpha$-tocopherol levels increased significantly following a triathlon race (5). We suggested that the mobilization of vitamin $\mathrm{E}$ from adipose tissues might be enhanced due to an increase in lipolysis caused by prolonged exercise.

Before vitamin E supplementation, the serum- $\alpha$-tocopherol level did not change after exercise, while the RBC- $\alpha$-tocopherol level decreased significantly. The decrease of the vitamin E level in RBC suggests the consumption of vitamin $\mathrm{E}$ to protect the RBC from oxidative damage caused by ROS during exercise. Therefore, we investigated the effect of vitamin $\mathrm{E}$ supplementation on serum- and RBC- $\alpha$-tocopherol levels before and after exercise in subjects with a normal vitamin $\mathrm{E}$ intake from daily foods because we found that serum- and RBC- $\alpha$-tocopherol levels were decreased by exercise.

After vitamin E supplementation, the serum- $\alpha$-tocopherol level showed a significant decrease after exercise; however, RBC- $\alpha$-tocopherol did not change after exercise. These results suggest that when there is a sufficient amount of vitamin $\mathrm{E}$ in the serum of subjects at the time of exercise, vitamin E may be shifted from the serum to the RBC by a gradient of vitamin E concentration. However, the details of this mechanism are not clear. Since the RBC- $\alpha$-tocopherol level was maintained after exercise, vitamin E consumed in RBC was possibly supplemented by vitamin E present in the serum. Furthermore, there was a significant negative correlation between the change in serum- $\alpha$-tocopherol level and the change in RBC- $\alpha$-tocopherol level only after vitamin E supplementation. This data also supports the movement of vitamin $\mathrm{E}$ from the serum to RBC during exercise when vitamin E levels in the serum are high. It can be suggested that a new supply of vitamin $\mathrm{E}$ from the serum to RBC is needed to replenish vitamin E consumed in RBC in order to maintain a certain RBC- $\alpha$-tocopherol level to protect cells against oxidative damage. Furthermore, it is necessary to investigate the details of the inter- and intra-variation of the $\alpha$-tocopherol level in serum and RBC during exercise.

In conclusion, vitamin $\mathrm{E}$ in $\mathrm{RBC}$ may be consumed to protect the cell membrane against oxidative damage during exercise. A subsequent movement of vitamin $\mathrm{E}$ from the serum to RBC seems to occur in order to prevent oxidative damage during exercise. From these results, we propose that it is crucial to have a sufficient supply of antioxidants such as vitamin E during exercise.

\section{Acknowledgments}

We give our thanks to the subjects who participated in this study and Ms. Young M. Park and Mr. Toshio Kimura for their help in the preparation of the manuscript.

Vitamin E supplement used in this study was provided by Eisai Co. Ltd.

\section{REFERENCES}

1) Alessio HM. 1993. Exercise-induced oxidative stress. Med Sci Sports Exerc 25: 218-224.

2) Szczesniak L, Karolkiewicz J, Deskur E, Rychlewski T, Konys L, Stankiewicz K. 1998. The influence of exercise-induced oxidative stress on binding and degradation of ${ }^{125}$ I-insulin by the receptors on erythrocytes. $J$ 
Physiol Pharmacol 49: 421-432.

3) Oostenbrug GS, Mensink RP, Hardeman MR, De Vries T, Brouns F, Hornstra G. 1997. Exercise performance, red blood cell deformability, and lipid peroxidation: effects of fish oil and vitamin E. J Appl Physiol 83: 746-752.

4) Packer L. 1991. Protective role of vitamin E in biological systems. Am J Clin Nutr 53: 1050-1055.

5) Kawai Y, Iwane H, Takanami Y, Shimomitsu T, Katsumura T, Fujinami J. 1994. Vitamin E is mobilized in relation to lipolysis after strenuous endurance exercise. Med Sci Sports Exerc 26: S7.

6) Pincemail J, Deby C, Camus G, Pirnay F, Bouchez R, Massaux L, Goutier R. 1988. Tocopherol mobilization during intensive exercise. Eur J Appl Physiol 57: 189191.

7) Misra HP, Fridovich I. 1972. The generation of superoxide radical during the autoxidation of hemoglobin. $J$ Biol Chem 247: 6960-6962.

8) Richmond W. 1973. Preparation and properties of a cholesterol oxidase from Nocardia sp. and its application to the enzymatic assay of total cholesterol in serum. Clin Chem 19: 1350-1356.

9) Abe K, Katsui G. 1975. Determination of tocopherols in serum by high-speed liquid chromatography. Vitamins (Japan) 49: 259-263.
10) Hatam LJ, Kaydan HJ. 1979. A high-performance liquid chromatographic method for the determination of tocopherol in plasma and cellular elements of the blood. $J$ Lipid Res 20: 639-645.

11) Dill DB, Costill DL. 1974. Calculation of percentage changes in volumes of blood, plasma, and red cells in dehydration. J Appl Physiol 37: 247-248.

12) Loschen S, Azzi A, Flohe L. 1973. Mitochondrial $\mathrm{H}_{2} \mathrm{O}_{2}$ formation: Relationship with energy conservation. FEBS Lett 33: 84-87.

13) Lindsay T, Romaschin A, Walker PM. 1989. Free radical mediated damage in skeletal muscle. Microcirc Endothelium Lymphatics 5: 157-170.

14) Sumida S, Tanaka K, Kitao H, Nakadomo F. 1989. Exercise-induced lipid peroxidation and leakage of enzymes before and after vitamin $\mathrm{E}$ supplementation. Int $J$ Biochem 21: 835-838.

15) Kanter MM, Nolte LA, Holloszy JO. 1993. Effects of an antioxidant vitamin mixture on lipid peroxidation at rest and postexercise. J Appl Physiol 74: 965-969.

16) Duthie GG, Rabertson JD, Maughan RJ, Morrice PC. 1990. Blood antioxidant status and erythrocyte lipid peroxidation following distance running. Arch Biochem Biophys 282: 78-83. 